\title{
Influence of Single and Double Membrane Roofs on Thermal Behaviour of Enclosed Space
}

\author{
Dragan KOSTIC, Vuk MILOSEVIC, Veliborka BOGDANOVIC, Miomir VASOV, Aleksandar VUCUR
}

\begin{abstract}
Even though membrane structures have been increasingly used in the world, their energy efficiency is very unfavourable due to poor thermal properties. This causes relatively high consumption of heating energy in the winter, as well energy consumed for the operation of air-conditioning during the summer, that is, under circumstances which require thermal comfort. Since thermal comfort depends on the air temperature and temperature of the interior surfaces of the envelope, this paper presents a study of the measurement and comparison of temperatures of single and double membrane structures in the summer period. Data collection was accomplished by measuring two adjacent structures, one covered with single and the other covered by a double membrane. Verification of the measured data was performed by comparing it with the official measured temperatures in the same period. The air temperatures were measured in all the separate air compartments and particular surfaces of each membrane. By analysing the measured data and their classification based on daylight and night-time periods, certain conclusions were drawn about the influence of an additional layer of membrane on the temperature of the air inside a covered structure.
\end{abstract}

Keywords: air temperature; energy efficiency; fabric structures; membrane structures; single and double membranes; surface temperature; textile membranes; thermal properties

\section{INTRODUCTION}

Contemporary membrane structures have actively been used in the world since the mid- $20^{\text {th }}$ century. Their form is double curved and they are characterized by their extremely low weight, which is the consequence of their thinness [1,2]. Even though their thickness of around $1 \mathrm{~mm}$ is an advantage in the structural sense, it represents a big problem in the thermal protection of space covered by membrane structures. Nowadays, membranes are mostly used for covering open spaces where they provide protection from the rain, sun, wind and snow, while their use for enclosed structures is characterized as energy inefficient due to poor thermal properties.

Thermal properties of membrane structures are still relatively unexplored. He and Hoyano studied the thermal properties of single-layered open membranes [3, 4]. Closed and semi-closed membranes were researched by Elnokaly, Chilton and Wilson [5] from a thermal optical aspect. The potential for implementation of CFD (computational fluid dynamics) analysis on open single-layered membranes in the Fluent software was tested by Elnokaly [6]. The thermal characteristics of closed membranes were dealt with by Devulder, Wilson and Chilton [7]. The most thorough research of thermal properties of membranes was provided by Harvie in his doctoral dissertation [8]. Researchers tend to improve the thermal protection of these structures in various ways. One of the proposals is the addition of layers of thermal insulation on the single membrane [9]. However, this has a negative impact on other properties of the membrane, primarily on its transparency.

Poor thermal properties cause a relatively high consumption of heating energy in the winter period, as well as energy consumed for the operation of air-conditioning during the summer, under the circumstances of required thermal comfort. Since thermal comfort depends on the temperatures of the air and internal surfaces of the skin enveloping certain space, this paper presents a study dealing with the measurement and comparison of air temperatures and of surfaces of single and double membranes. Double membranes appeared as an attempt to improve the thermal characteristics of membrane structures, since the formation of multiple partitions is one of the most common ways to improve the thermal protection of structures [10]. For this reason, it is expected that a double membrane will have better thermal characteristics. Nevertheless, according to the knowledge available to the authors of this paper, comparative measurements of single and double membranes have not been performed in the way presented in this research. The goal of this paper is the quantification of advantages of double membranes through the presentation of differences in temperatures of the air and of the surfaces in the halls covered by single and double membrane structures that have cylindrical shape.

\section{RESEARCH DESCRIPTION 2.1 Analysed Building}

For the needs of this research, one characteristic structure was selected, and all measurements taken were of this structure. It is located in Belgrade, in Grčića Milenka street, no. 74. On the east side of the building, there is a park, while housing buildings are located on the other sides. The reason for selecting this building as the most fitting is because it is composed of two halls, one of them covered with a single, and the other with a double membrane. The layout dimensions of each of the halls are $24 \times 44 \mathrm{~m}$, and its height is $11 \mathrm{~m}$. The shape of the halls is cylindrical, and the supporting structure is made of arches of glued-laminated timber $70 \mathrm{~cm}$ high. The implemented membrane structure has a thickness of $1 \mathrm{~mm}$, with a bearing microfiber core and PVC protection (Membrane Type I). The single membrane is placed on the top side of the main arches, and in case of double membranes, one membrane is placed on the top side, and the other on the bottom side of the main arches. The air between the layers of the double membrane is enclosed. Comparative testing of these two halls was performed because of their numerous similarities, including shape, size, orientation and location.

However, there are certain differences, apart from the cover, which is in one case a single, and in the other a double membrane. There is a difference in floor materialization. In the case of the single membrane hall, the 
floor is covered with artificial grass, and in the case of the double membrane, it is a concrete floor with an epoxide surface. In addition, on the north side of the double membrane hall there is a longitudinal masonry wall 4 meters high. The hall interiors are presented in Fig. 1, and the orientation of the halls in Figs. 2 and 3.
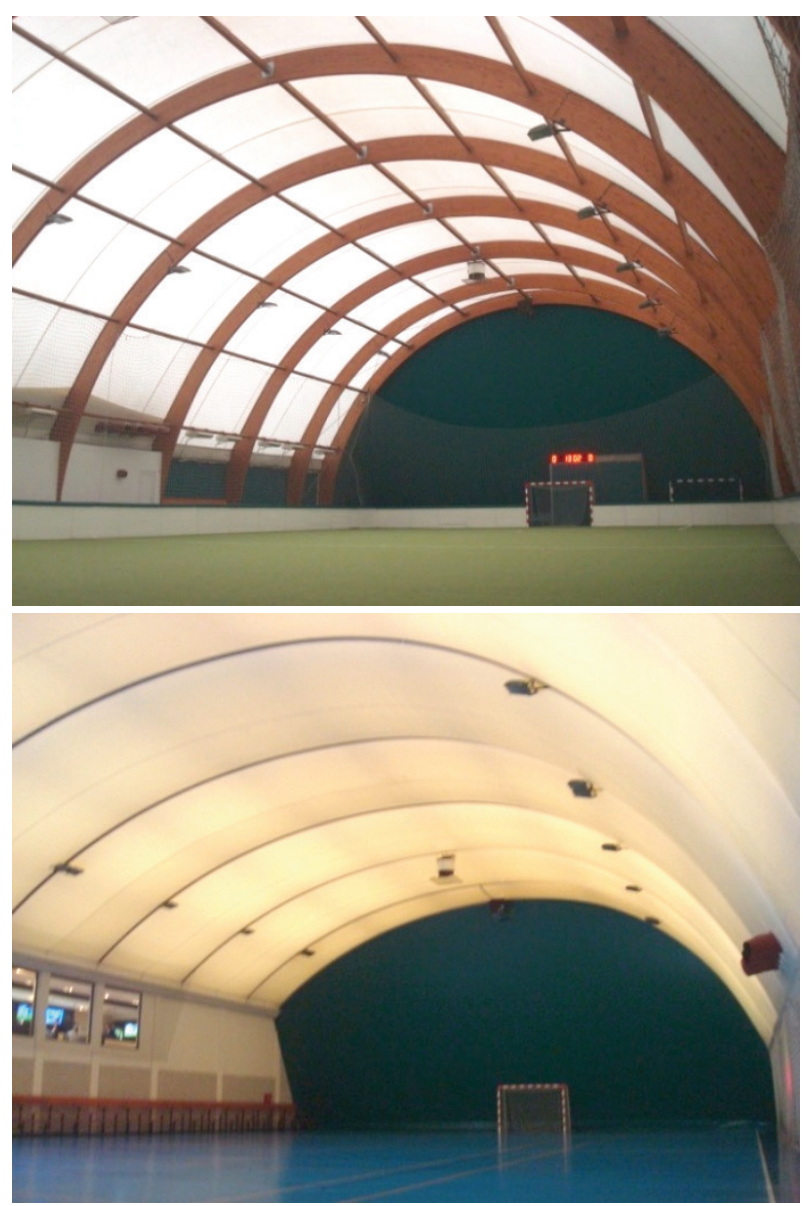

Figure 1 Analysed single and double membrane structures

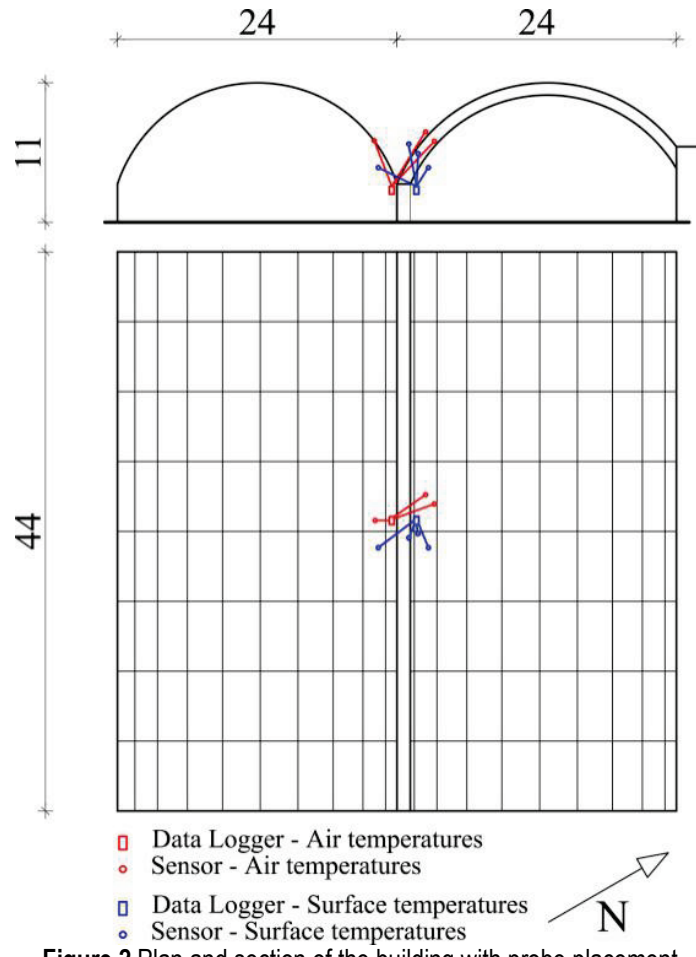

Figure 2 Plan and section of the building with probe placement

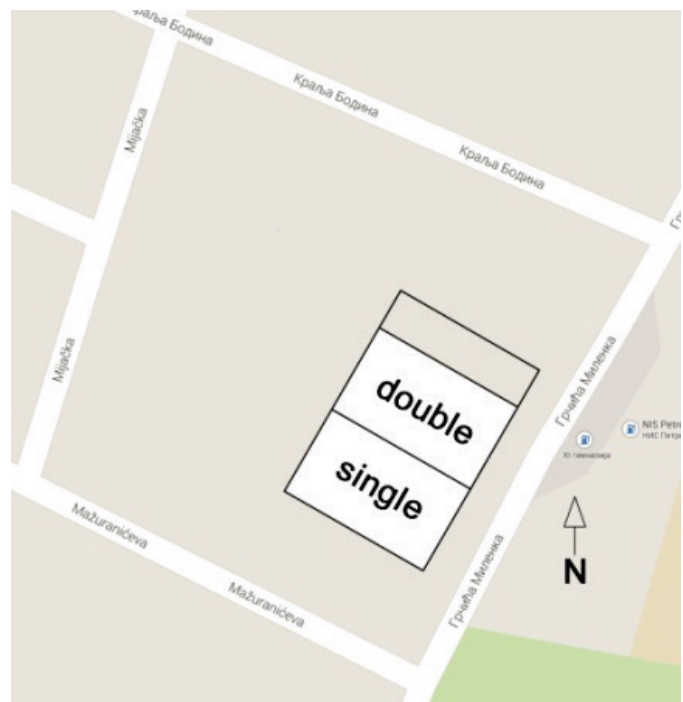

Figure 3 Aerial plan

\subsection{Research Methods}

The applied research methods include the collection of data on air temperatures and data on membrane surfaces temperatures obtained by measuring instruments in situ, a comparison of the values with official meteorological data, data analysis using mathematical methods of statistics and probability (analysing the differences between the measured and official data, and analysing the effects that led to rapid temperature changes). In such a way conclusions about the benefits of double compared to single membrane will be drawn.

\subsection{Measuring on Site and Equipment}

The air temperature and the temperature of the membrane surfaces were measured with the aid of resistance temperature detectors (RTDs). Those probes are equipped with sensors type Pt100, with resistance tolerances specified in DIN/IEC751 as Class A. Probes are PVC insulated with a working temperature range of -20 to $70{ }^{\circ} \mathrm{C}$ and may be used as multifunctional probes to measure gas stream, liquid or surface temperature. The proposed use of the probe depended on the configuration of the sensor through the data logger procedure. In this particular case, we used the Data Logger KTR 300 KISTOCK produced by Kimo Co. France (Fig. 6a). The apparatus was mounted for a full 14 days from June 20, 2014, until July 5, 2014, whereby the data were recorded at 30-minute intervals. The probes for surfaces and air temperatures were fitted on the building as in Fig. 2, Fig. $6 \mathrm{~b}$ and Fig. 6c. One probe for measuring the surface temperature was fitted on the external and one on the internal layer of the double membrane, and one on the single membrane. Air temperature probes were fitted so that they measure external air temperatures, air between the two layers of the double membrane, the internal air below the single membrane and the internal air below the double membrane. This setup was configured like the air temperature measurements in Harvie's pilot studies [8].

\subsection{Official air Temperatures}

Apart from the measuring in situ, the official data on the weather conditions in the analysed period were also 
used. These data were taken from two different sources. The first source was the web site www.ogimet.com. The station WMO 13274 was chosen as the most suitable one because of its proximity. It is at a distance of $2 \mathrm{~km}$ northeast from the building location. It is an automatic measuring station which records data at three-hour intervals. The second source was the website www.wunderground.com. The measuring station in this measuring system is called ISRBIJAB2, and it collects data every 5 minutes. The distance from the station is around $2,5 \mathrm{~km}$, north-east of the structure location.

Comparison of the measured air temperature and the official meteorological data in the analysed period pointed to the validity of the measured values.

\section{$3 \quad$ RESULTS AND DISCUSSION}

The results are provided together with their discussion, divided into subchapters. These sub-chapters include official weather data and their comparison with the external temperatures measured in situ (Fig. 4 and Fig. 5), as well as collected measuring results of membrane surfaces (Fig. 8). The results recorded using the Data Logger were firstly displayed in diagrams, and then their characteristic average and extreme values were provided in Tables for easy comparison (Tabs. 1 and 2). All the results were separately analysed and discussed, and in the conclusion, the most important conclusions based on the discussion presented in this chapter are shown.

\subsection{Official and Recorded Outside Air Temperatures}

The diagram in Fig. 4 represents a detailed record of the official measured air temperatures from the stations WMO13274 and ISRBIJAB2, and measured temperatures of the outside air above the structure.
The displayed diagram shows that the official measured air temperatures from the two measuring stations are very similar but that they do not fully coincide. This phenomenon is expected because the stations are at a mutual distance of around $3 \mathrm{~km}$, so it is natural that the natural conditions around them are not completely identical. The relationship of the official measured temperatures and the temperature of the outside air above the structure should be explained additionally, because it is obvious that there are discrepancies between these temperatures, especially during daylight. During daylight, all of the temperatures reach their maximum at the same time, but the temperature of the outside air above the hall is significantly higher than at the measuring stations. It should, however, be born in mind that the official measurements abide by certain rules which cannot be adhered to when measuring temperatures on the structures. These rules comprise protection of the measuring location from direct sunlight, wind, rain and other impacts which can temporarily affect the value of the measured temperature. In addition to this, and equally important, is that the official measuring stations are installed at locations with a vegetation-rich environment. In this manner, rapid micro-climatic variations in temperature are prevented, and more constant results are obtained. The measuring on the structure took place in a completely different environment. This, however, provides a much more realistic picture of what the actual micro-climate conditions were at the microlocation. The results of the measured temperatures clearly show external influences acting on the measuring location. The measured temperature of the outside air above the structure has multiple short-term oscillations, and reaches higher maximum temperatures, but in general, it follows the periods of the official temperatures.

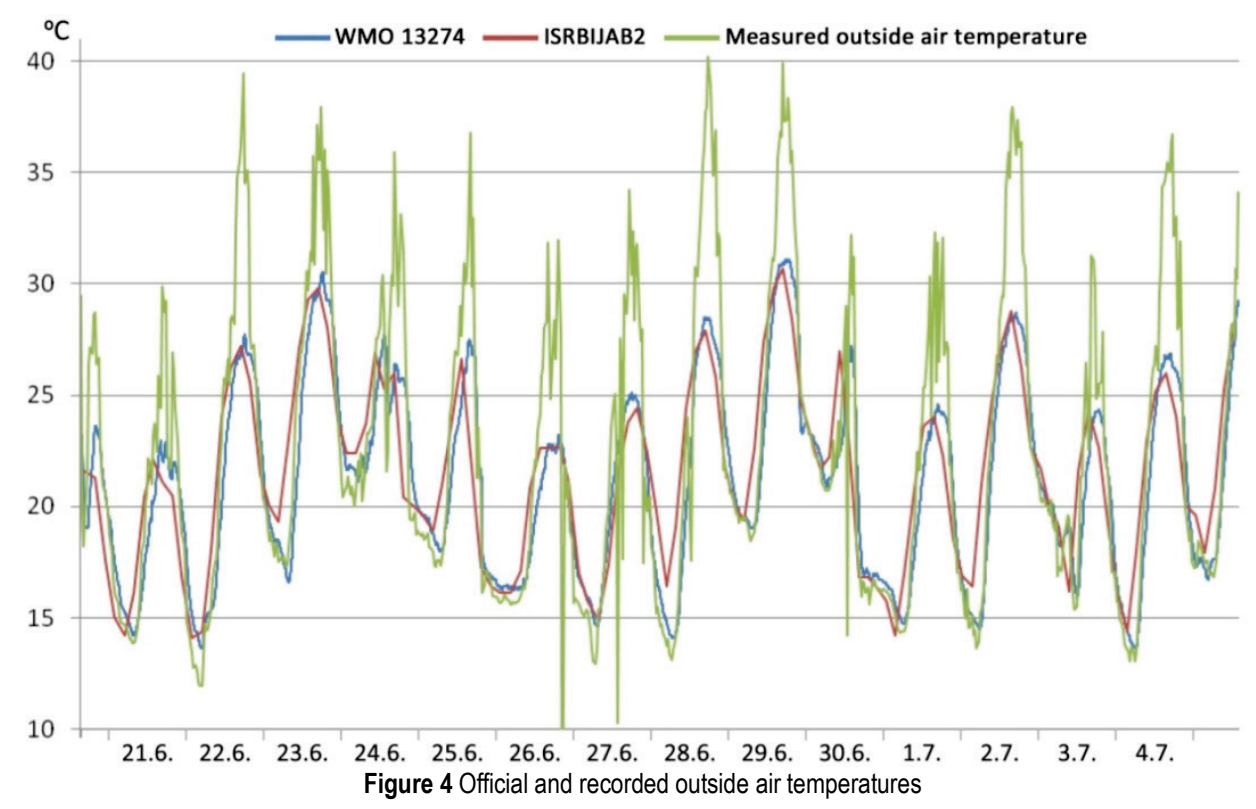

During the night, when there is no sunlight, the official temperatures and the temperature of the outside air measured above the structure are almost equal, but it is noticeable that the air temperature above the structure is then slightly lower. Yet, the fact that at night these temperatures are close, and the fact that both the measured and official temperatures follow the same periods of oscillations and that they reach the minimum and maximum temperatures at the same time is an indicator verifying the accuracy of the performed measurements. 
The irregularities occurring on the diagram of the measured outside temperatures on the structure will be explained in detail in the next subchapter, together with other measured temperatures on the structure.

\subsection{Recorded Air Temperatures}

The air temperatures on the structure, recorded by measuring, are provided by the diagram in Figure 5, where:

- $\theta_{1}^{\mathrm{A}}-$ the air temperature in the hall covered with a single membrane

- $\theta_{2}{ }^{\mathrm{A}}$ - the air temperature in the hall covered with a double membrane

- $\theta_{\mathrm{i}}^{\mathrm{A}}-$ the air temperature between the layers of the double membrane

- $\theta_{\mathrm{o}}{ }^{\mathrm{A}}-$ the air temperature of the outside air above the hall
In the air temperatures diagram, several regularities can be observed. The oscillations of all the measured temperatures are approximately sinusoidal, and the period is equal to one day, so each day there is a temperature maximum and minimum. For this reason, the diagram can be divided into intervals of one day, and they can be studied separately. By comparing all the measured air temperatures during each individual day, we draw a conclusion that they are in the same mutual relationship, but that their intensities differ from day to day. Each day can be divided into daylight and night, and in these two periods of time the mutual relationships of temperatures are completely different. The diagrams of the measured air temperatures during one characteristic period of daylight and night-time are shown in Fig. 7.

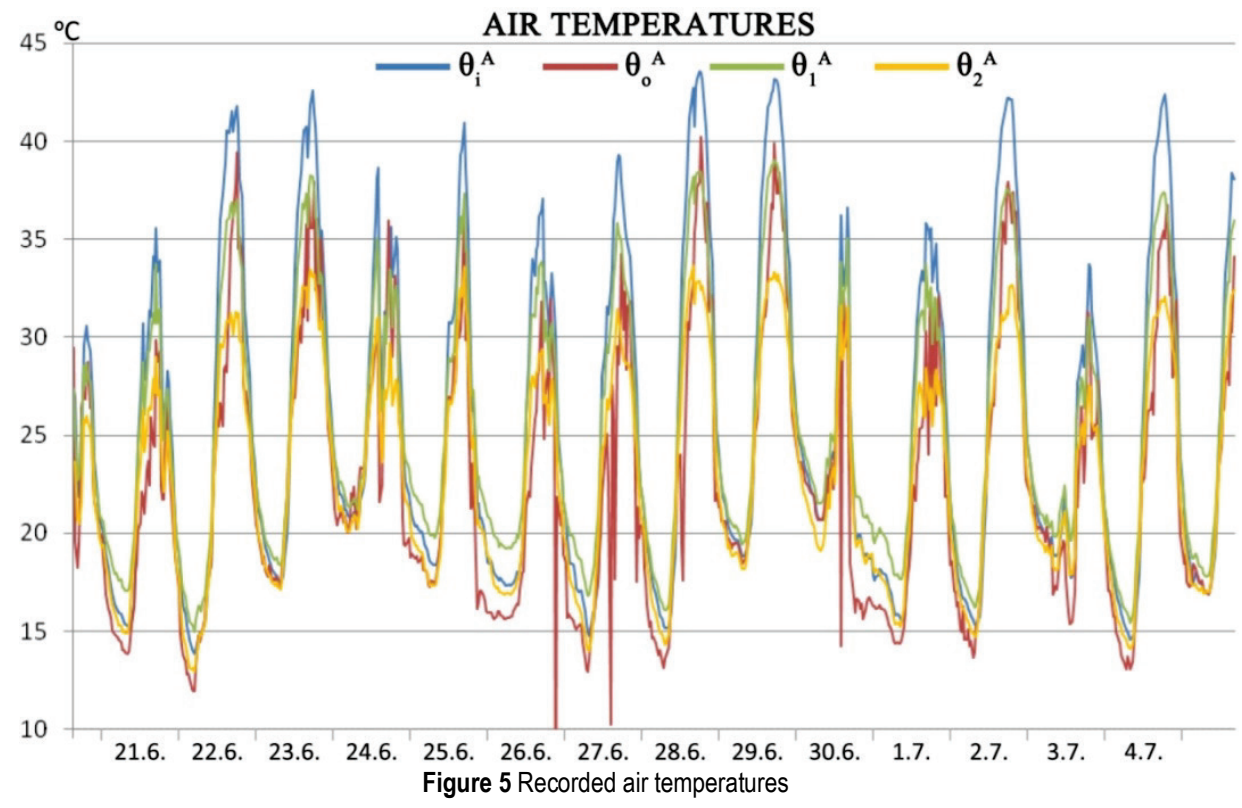

a.

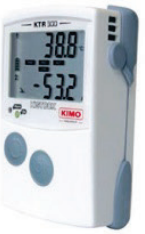

b.

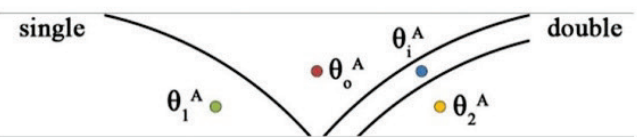

c.

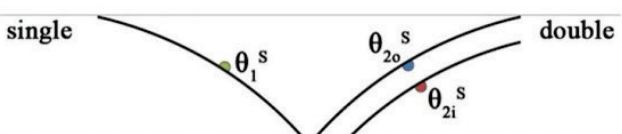

Figure 6 a) Datalogger with temperature wire probe, b) Approximate illustrations of air temperature probe's positions, c) Approximate illustrations of surface temperature probe's positions

By reading the results from the given diagrams, the following observations can be made:

- The air temperature between the layers of the double membrane is the highest during daylight

- The air temperature below the single membrane during daylight is higher than the external temperature

- The air temperature below the double membrane is the lowest during daylight

- At night, the relationships are different, and the outside air has the lowest temperatures. The air below the double membrane has a slightly higher temperature, followed by slightly higher temperature of the air between the layers of the double membrane, while the air below the single membrane has the highest temperature
The analysis of these results provides an explanation of why temperatures have such relationships during daylight.

- The air temperature between the layers of the double membrane $\theta_{\mathrm{i}}^{\mathrm{A}}$ is the highest because the air is enclosed between two membranes, and has the lowest volume. As a result of solar radiation and of the increase of external temperature, it heats up and reaches the highest temperatures.

- The air temperature $\theta_{1}^{\mathrm{A}}$ is until afternoon higher than the outside air temperature $\theta_{\mathrm{o}}{ }^{\mathrm{A}}$, and then it follows the temperature drop $\theta_{0}{ }^{\mathrm{A}}$

- The temperature $\theta_{2}{ }^{\mathrm{A}}$ is the lowest of all air temperatures during daylight. It approximately follows the temperature increase of the outside air $\theta_{\mathrm{o}}{ }^{\mathrm{A}}$ until 
noon, but by night-time, it becomes significantly lower than all the other air temperatures because it is protected from the solar radiation by the double membrane.

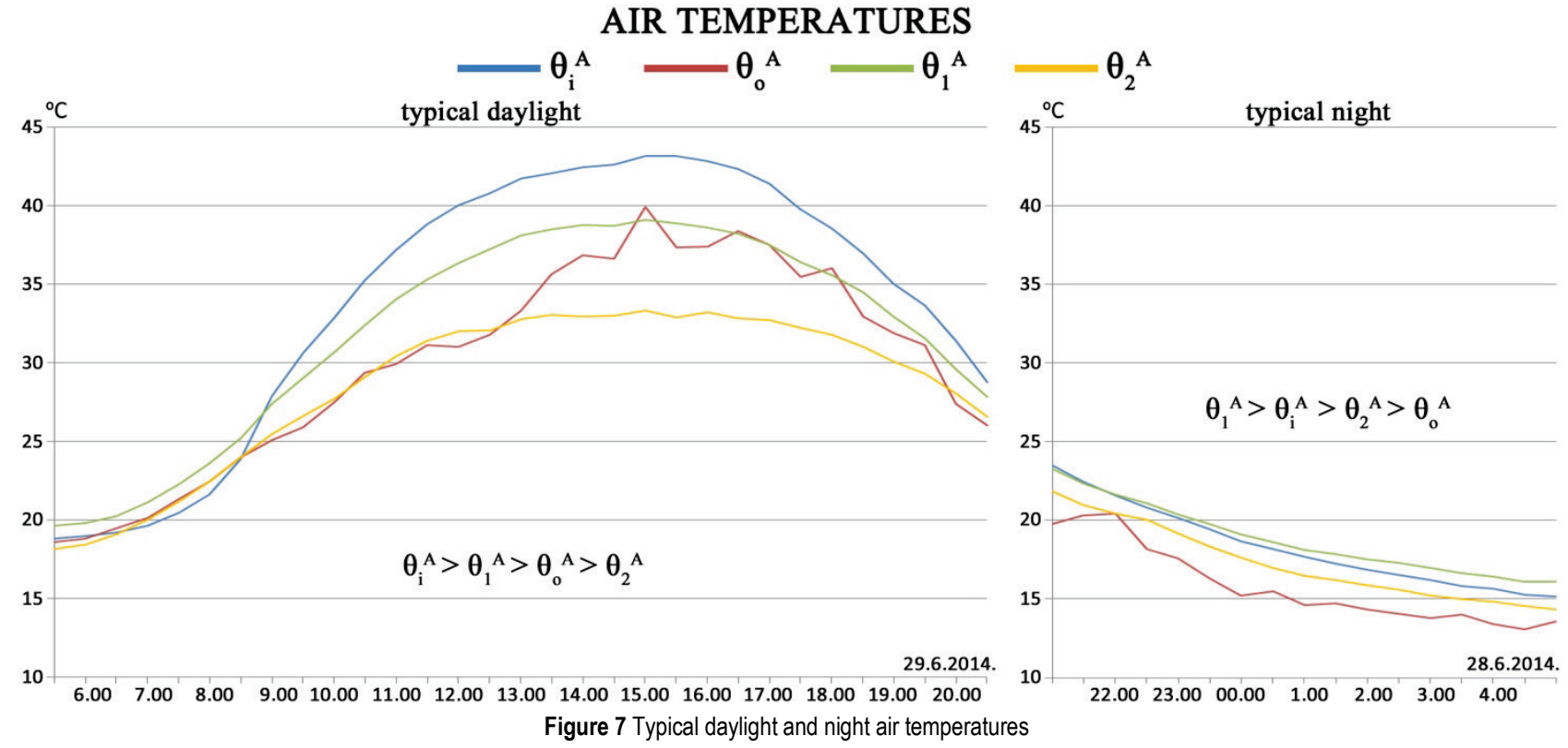

At night:

- The outside air $\theta_{\mathrm{o}}{ }^{\mathrm{A}}$, has the lowest temperature, because the halls retain a certain amount of heat.

- The temperature of the higher layers of air inside the hall is higher, because the warmer air has a lower density, and tends to rise high. Because of this $\theta_{\mathrm{i}}{ }^{\mathrm{A}}$ is higher than $\theta_{2}{ }^{\mathrm{A}}$. This difference is not large, but it is noticeable and always present.

- Eventually, even though it was not expected, the results indicate that at night $\theta_{1}{ }^{\mathrm{A}}$ is higher than all the other temperatures. At first sight, this appears illogical, because the double membrane should be a better insulator than the single membrane and should retain more heat. However, during daylight, $\theta_{1}{ }^{\mathrm{A}}$ is significantly higher than $\theta_{2}{ }^{\mathrm{A}}$ so even though the double membrane is better insulation, the air below the single membrane at night does not lose enough heat to become cooler than the air below the double membrane. On average, temperature $\theta_{1}{ }^{\mathrm{A}}$ during the night drops by $8.16^{\circ} \mathrm{C}$ compared to the daylight and $\theta_{2}{ }^{\mathrm{A}}$ by $7.11{ }^{\circ} \mathrm{C}$, which confirms that the double membrane is a better insulation.

It can be concluded that all the mentioned relationships of air temperatures in halls 1 and 2 are primarily a consequence of better thermal characteristics of the double membrane, but also of small influences of the different floors in the halls and the presence of the longitudinal wall in the hall covered by a double membrane.

It is important to say that there are several places on the air temperature diagram, which appear to be anomalies. However, for every one of them, there is an explanation. These anomalies are mostly reflected in drastic air temperature drops, or considerable drops of outside air temperature. As early as on the first day of measuring, on June 20, 2014 in the period when the highest daylight temperatures are expected, the air temperature drop of over $5{ }^{\circ} \mathrm{C}$ on average was observed. By comparing this to the official data from the web site www.wunderground.com, this drop of measured temperatures can easily be explained. Namely, exactly in this period, the measuring station ISRBIJAB2 recorded a short episode of rain. A similar situation was recorded the following day when during daylight, the measured temperatures on the structure dropped, but when the official weather data also exhibited an unexpected temperature drop, and later on, its increase. On June 24, 2014, there was another drop, the largest one of the measured air temperature drops, but once again it was caused by the drop in the outside temperature and light rain, which was confirmed by the official air temperature data. Drastic outside air temperature drops were recorded on June 26, 27 and 30, 2014. These drops were recorded only in one measurement each. There were no considerable variations in other air temperatures, precisely because the external air temperature variation was short-term. However, the possibility of a malfunction or error of the measuring equipment is excluded by the fact that the drop was recorded in surface temperatures, primarily of the single membrane surfaces, which are most susceptible to variation because of the low thermal mass [5]. In addition, Devulder in his paper [7] mentions a recorded temperature drop of over $5^{\circ} \mathrm{C}$, in the course of only one minute, which is obviously a phenomenon, which is not unusual.

Tab. 1 indicates that the results obtained by measuring could be better quantified. Individual air temperature measurements were not shown in Table 1 for reasons of clarity, because there are over 4000 recorded values. It was decided that the best thing to do is to present the average and extreme values of the measured temperatures and their mutual relationships. This way is the simplest one for drawing general conclusions, since the values are more useful than the data concerning individual moments in time. The methodology described in the previous section of the result analysis was repeated, so each day was divided into daylight and night, as the two characteristic periods. The analysed period starts with the beginning of June 21 until the end of the July 4 . In the measurement period, the 
average duration of the sunrise was $4.54 \mathrm{~h}$, and of the sunset $20.28 \mathrm{~h}$. Three individual measurements recorded on June 26, 27 and 30, mentioned in the previous paragraph were excluded due to the extreme external conditions at those times. In Tab. 1, in first four columns provided the measured air temperatures, and in the following six columns the mutual differences of those temperatures. In the rows, the following data were provided in this order: average, lowest and highest value during daylight, then average, lowest and highest value at night, and eventually, the lowest and highest value during the entire period, including day and night. The results summed up once again confirm the conclusions from the previous part of this chapter, when the results were descriptively analysed and assessed.
- During daylight, the average air temperature between the layers of the double membrane is $29,46{ }^{\circ} \mathrm{C}$, and it is $4,00{ }^{\circ} \mathrm{C}$ higher than the outside temperature, $1,23^{\circ} \mathrm{C}$ higher than the temperature below the single membrane and $4,05{ }^{\circ} \mathrm{C}$ higher than the temperature below the double membrane.

- During the night, the temperature below the single membrane is the highest, $20,07{ }^{\circ} \mathrm{C}$ on average, followed by the air temperature of the enclosed space between the layers of the double membrane of 19,22 ${ }^{\circ} \mathrm{C}$, the air temperature below the double membrane of $18,30^{\circ} \mathrm{C}$, and the lowest is the outside temperature at $17,64^{\circ} \mathrm{C}$

Table 1 Air temperatures

\begin{tabular}{|c|c|c|c|c|c|c|c|c|c|c|}
\hline${ }^{\circ} \mathrm{C}$ & $\theta_{\mathrm{i}}^{\mathrm{A}}$ & $\theta_{\mathrm{o}}^{\mathrm{A}}$ & $\theta_{1}^{\mathrm{A}}$ & $\theta_{2}^{\mathrm{A}}$ & $\theta_{\mathrm{i}}^{\mathrm{A}}-\theta_{\mathrm{o}}^{\mathrm{A}}$ & $\theta_{\mathrm{i}}^{\mathrm{A}}-\theta_{1} \mathrm{~A}$ & $\theta_{\mathrm{i}}^{\mathrm{A}}-\theta_{2}^{\mathrm{A}}$ & $\theta_{1}^{\mathrm{A}}-\theta_{\mathrm{o}}^{\mathrm{A}}$ & $\theta_{2}^{\mathrm{A}}-\theta_{\mathrm{o}}^{\mathrm{A}}$ & $\theta_{1}^{\mathrm{A}}-\theta_{2}^{\mathrm{A}}$ \\
\hline Average during daylight & 29.46 & 25.46 & 28.23 & 25.41 & 4.00 & 1.23 & 4.05 & 2.77 & -0.05 & 2.82 \\
\hline Minimum during daylight & 14.54 & 13.05 & 15.45 & 13.96 & -3.06 & -3.44 & -2.33 & -3.79 & -8.42 & 0.53 \\
\hline Maximum during daylight & 43.57 & 40.22 & 39.05 & 33.68 & 18.88 & 5.25 & 11.14 & 16.04 & 11.86 & 6.32 \\
\hline Average at night & 19.22 & 17.64 & 20.07 & 18.30 & 1.57 & -0.86 & 0.91 & 2.43 & 0.66 & 1.77 \\
\hline Minimum at night & 13.82 & 11.94 & 14.99 & 12.95 & -1.34 & -2.32 & -0.47 & -0.62 & -1.63 & 0.71 \\
\hline Maximum at night & 27.21 & 25.01 & 26.75 & 25.52 & 4.07 & 0.74 & 2.40 & 5.10 & 3.20 & 2.78 \\
\hline Minimum & 13.82 & 11.94 & 14.99 & 12.95 & -3.06 & -3.44 & -2.33 & -3.79 & -8.42 & 0.53 \\
\hline Maximum & 43.57 & 40.22 & 39.05 & 33.68 & 18.88 & 5.25 & 11.14 & 16.04 & 11.86 & 6.32 \\
\hline
\end{tabular}

From Tab. 1 it is possible to read other values related to the highest and lowest recorded temperatures during daylight and night, and the values correlated with the mutual relationships of the temperatures, but they will not be individually commented upon. The most significant piece of data in Tab. 1, considering the topic and goal of the research, and probably the most important piece of data of the entire paper, is the value in the first row and the last column. This value indicates that during daytime, the temperature of the air in the hall covered by the single membrane is $2,82{ }^{\circ} \mathrm{C}$ higher on average than the air temperature in the hall covered by the double membrane. This data refers to the summer period, i.e. to the situation when the outside temperature is considerably higher than the interior one. Precisely this data illustrates the relationship of the thermal protection offered by the double membrane as opposed to the single membrane, and it confirms that the double membrane offers considerably better thermal protection.

\subsection{Recorded Surface Temperatures}

Surface temperatures were given in the diagram in Fig. 8, where:

- $\theta_{1}{ }^{\mathrm{S}}-$ the temperature of the outside surface of the single membrane

- $\theta_{20} \mathrm{~S}$ - the temperature of the interior surface of the external layer of the double membrane

- $\theta_{2 \mathrm{i}}^{\mathrm{S}}$ - the temperature of the interior surface of the interior layer of the double membrane

The diagram of surface temperatures, similar to the one of air temperatures, exhibits certain regularities. In this diagram, as well the temperature oscillations are approximately sinusoidal, within the period of a single day. For this reason, this diagram can be divided into days as well, and the periods of daylight and night can be separately observed (Fig. 9). During these periods, the surface temperatures show different behaviour and have different mutual relationships. It is important to note that an undesirable event took place on July 1, 2014, when the surface temperature probe of the hall covered by a single membrane was mistakenly removed by an employee of the structure. For this reason, there are no valid data on the values of the temperature for the last four days. Yet, the measured values of this temperature in the preceding 11 days show sufficient regularities so that the conclusions about its behaviour can be drawn, and it can be assumed with considerable certainty what the values would have been had the mentioned unforeseeable event not taken place.

During daylight $\theta_{1} \mathrm{~S}$ is drastically higher than the other surface temperatures. Closely following it is $\theta_{20} \mathrm{~s}$, while $\theta_{2 \mathrm{i}} \mathrm{S}$ is slightly lower than it. During the night, these relationships are reversed, $\theta_{1} \mathrm{~S}$ is the lowest, followed by $\theta_{20} \mathrm{~S}$, while the highest is $\theta_{2 \mathrm{i}} \mathrm{S}$. During the night, the values of these temperatures are mutually much closer than during daylight. Especially prominent among the results is the high temperature of the single membrane surface during daylight. The reason for it being so drastically higher than the other temperatures was that the probe for it was placed on the external side of the membrane. The most favourable situation would be if on any of the 3 existing membranes, 2 surface temperature probes would be fitted, one on the external and the other on the internal side. Then it would be possible to monitor the passage of heat through each of these membranes. However, this was not technically possible, since there were no additional probes available. Such measurement will be a part of further research which will be conducted as soon as the necessary conditions have been satisfied. In order to get at least a general view of what the difference between the external and internal surface of the membrane is, it was decided to place the probe 
externally on the single membrane, and to place another on the interior side of the external layer of the double membrane. The results indicated that during daylight, the side exposed to the sun has considerably higher temperature than the side which is not exposed. Even though those were not the sides of the same membrane, the difference is so high that it urges further investigation on this topic. At night, $\theta_{1} \mathrm{~S}$ is the lowest, because the probe is placed on the external side, so it is the most exposed to the cool outside air. Temperatures $\theta_{20} \mathrm{~s}$ and $\theta_{2 \mathrm{i}}^{\mathrm{S}}$ show the expected results. It is obvious that the double membrane offers certain thermal isolation, so during daylight, the internal layer is protected from the outside heat and has a lower temperature, and at night, it retains heat inside and has a higher temperature than the external one.
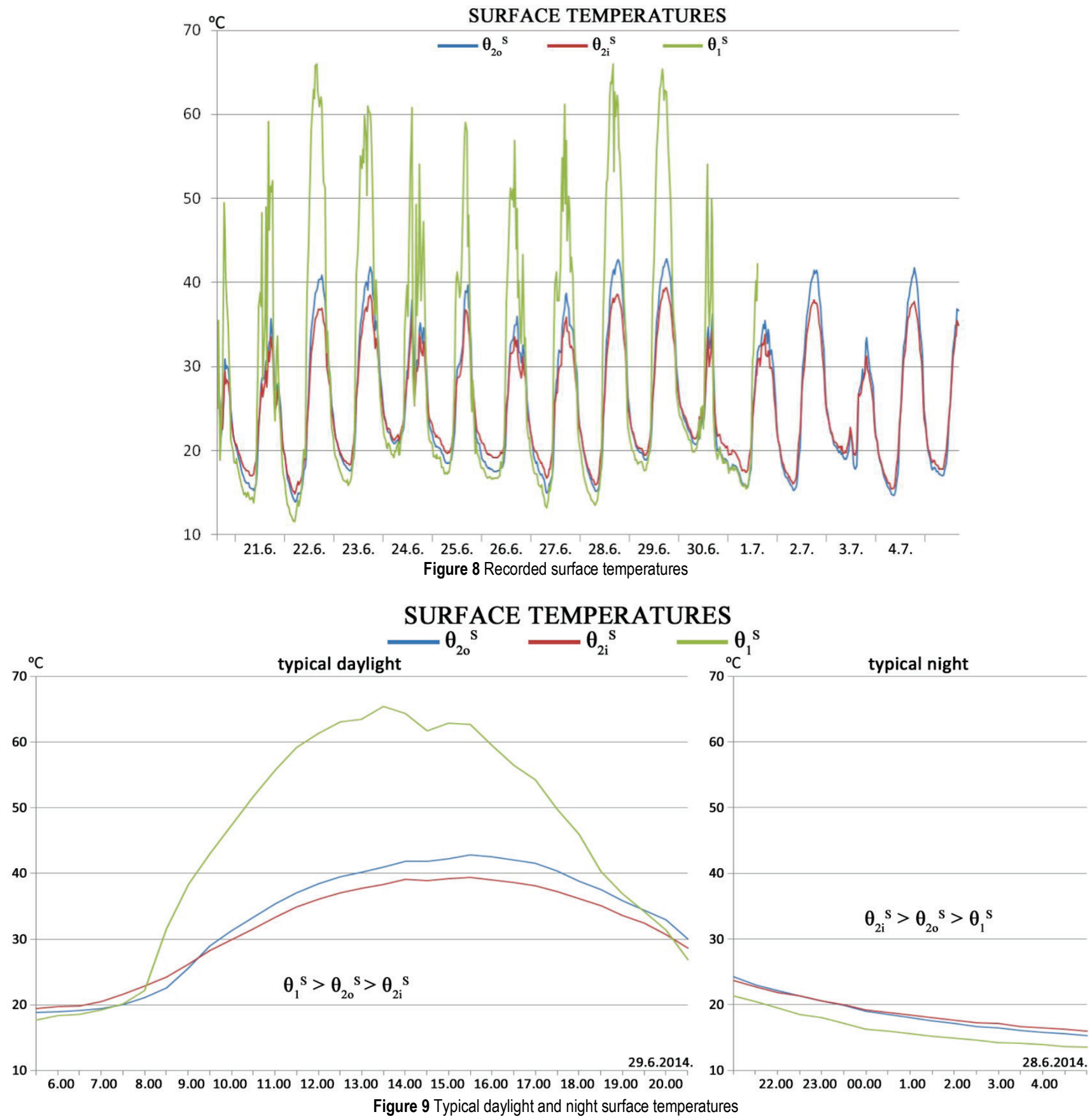

Tab. 2 is organized in a similar way as Table 1 in the previous subchapter. It provides the average and extreme values of the measured temperatures of layers. Also analysed are the values obtained by measurements taken between June 21 and July 1. This time period is divided into daylight and night and the results of these two parts of the day are provided separately. The first three columns in
Tab. 2 show the temperatures of the layers on the structure, and the following three columns show the mutual differences of these temperatures. The rows provide the following information, in this order: average, lowest and highest values during daylight, then average, lowest and highest value at night, and eventually lowest and highest value during the entire period including daylight and night. 


\begin{tabular}{|c|c|c|c|c|c|c|}
\hline${ }^{\circ} \mathrm{C}$ & $\theta_{20} \mathrm{~s}$ & $\theta_{2 i} \mathrm{~s}$ & $\theta_{1} \mathrm{~s}$ & $\theta_{20} \mathrm{~S}-\theta_{2 i} \mathrm{~S}$ & $\theta_{1} \mathrm{~s}-\theta_{20} \mathrm{~s}$ & $\theta_{1} \mathrm{~s}-\theta_{2 \mathrm{i}} \mathrm{s}$ \\
\hline Average during daylight & 29.57 & 28.57 & 37.95 & 1.00 & 8.39 & 9.39 \\
\hline Minimum during daylight & 14.91 & 15.91 & 13.25 & -3.05 & -5.82 & -4.55 \\
\hline Maximum during daylight & 42.81 & 39.46 & 66.01 & 4.42 & 27.09 & 30.07 \\
\hline Average at night & 19.79 & 20.42 & 17.99 & -0.63 & -1.80 & -2.43 \\
\hline Minimum at night & 13.87 & 14.85 & 11.54 & -2.02 & -3.59 & -3.77 \\
\hline Maximum at night & 28.04 & 27.20 & 25.46 & 1.12 & -0.26 & -0.84 \\
\hline Minimum & 13.87 & 14.85 & 11.54 & -3.05 & -5.82 & -4.55 \\
\hline Maximum & 42.81 & 39.46 & 66.01 & 4.42 & 27.09 & 30.07 \\
\hline
\end{tabular}

- On average, during daylight, the temperature of the single membrane surface is the highest and amounts to $37,95{ }^{\circ} \mathrm{C}$, and at night, it is the lowest and it amounts to $17,99{ }^{\circ} \mathrm{C}$

- Extreme maximum and minimum temperatures are also recorded on this surface and they are $66,01^{\circ} \mathrm{C}$ and $11,54{ }^{\circ} \mathrm{C}$

- Temperatures of the external and internal layer of the double membrane are similar, given that during daylight the external layer has a temperature which is on average $1,00{ }^{\circ} \mathrm{C}$ higher, and at night, on average $0,63{ }^{\circ} \mathrm{C}$ lower.

- Temperatures of the internal layer of the double membrane have lower oscillation amplitudes, and they range between $14,85{ }^{\circ} \mathrm{C}$ to $39,46{ }^{\circ} \mathrm{C}$, and the temperature of the external layer is between $13,87^{\circ} \mathrm{C}$ and $42,81{ }^{\circ} \mathrm{C}$.

\section{CONCLUSION}

Even though they provide advanced structural characteristics and attractive forms, membrane structures are used in practice considerably less than traditional structural systems. Some of the reasons for this are their poor thermal properties. This property makes them less desirable for covering enclosed structures, because of the increased heating and air conditioning energy consumption. It is considered that the best way to improve the thermal properties of membranes is the formation of double, triple or multiple covers made of membrane material $[8,9,10]$.

The results of this study showed the advantages of the cylindrical shaped double membrane compared to the single one, which was expected. In numerical terms, in the summer period during daylight, the air temperature inside the hall covered by the double membrane is on average $2,82{ }^{\circ} \mathrm{C}$ (Tab. 1) lower than the air temperature inside the hall covered by the single membrane. This proved that the double membrane provided better heat protection in comparison with the single membrane. Another indicator confirming this conclusion is the fact that the maximum air temperature inside the hall covered by the double membrane is by more than $5{ }^{\circ} \mathrm{C}$ (Tab. 1) lower than the maximum air temperature inside the hall covered by the single membrane. Every day, with no exceptions, the maximum air temperature inside the hall covered by the double membrane was considerably lower than all other maximum air temperatures, which indicates better thermal protection because the oscillation amplitudes of this temperature are the lowest. The surface temperatures showed similar behaviour, as the interior layer of the double membrane has the lowest temperature oscillations, so it confirms the previous conclusions. In the case of the analysed double membrane, the air enclosed between the layers was $70 \mathrm{~cm}$ thick, while considerably better results are expected in cases when during the summer period, the inside air between the layers is allowed to circulate in a natural or artificial way, which should be investigated further.

\section{Acknowledgments}

The work reported in this paper is a part of the investigation within the research project TR 36043 and TR 36028 supported by the Ministry for Education, Science and Technology Development Republic of Serbia. This support is gratefully acknowledged.

\section{REFERENCES}

[1] Bridgens, B. \& Birchall, M. (2012). Form and Function: The Significance of Material Properties in the Design of Tensile Fabric Structures. Engineering Structures, 44, 1-12. https://doi.org/10.1016/j.engstruct.2012.05.044

[2] Berger, H. (1999). Form and Function of Tensile Structures for Permanent Buildings. Engineering Structures, 21, 669679. https://doi.org/10.1016/S0141-0296(98)00022-4

[3] He, J. \& Hoyano, A. (2009). Measurement and Simulation of the Thermal Environment in the Built Space under a Membrane Structure. Building and Environment, 44, 11191127. https://doi.org/10.1016/j.buildenv.2008.08.003

[4] He, J. \& Hoyano, A. (2010). Measurement and Evaluations of the Summer Microclimate in the Semi-enclosed Space under a Membrane Structure. Building and Environment, 45 230-242. https://doi.org/10.1016/j.buildenv.2009.06.006

[5] Elnokaly, A., Chilton, J., \& Wilson, R. (2003). Environmental Performance of Spaces Enclosed or Semienclosed by Fabric Membrane Structures. Textile Composites and Inflatable Structures / Barcelona, 171-176.

[6] Elnokaly, A. (2014). CFD Analysis of Tensile Conical Membrane Structures as Microclimate Modifiers in Hot Arid Regions. Civil Engineering and Architecture, 2(2), 92-102.

[7] Devulder, T., Wilson, R., \& Chilton J. (2015). The Thermal Behaviour of Buildings Incorporating Single Skin Tensile Membrane Structures. International Journal of Low Carbon Technologies, 2(2), 195-213. https://doi.org/10.1093/ijlct/2.2.195

[8] Harvie, G. N. (1995). An Investigation into the Thermal Behaviour of Spaces Enclosed By Fabric Membranes. Cardiff University of Wales, Cardiff.

[9] Karwath, M. (2011). Membrane Structures with Improved Thermal Properties. International Conference on Textile Composites and Inflatable Structures / Barcelona, 312-318.

[10] Forster, B.; Mollaert, M. (2004). European Design Guide for Tensile Surface Structures. Tensinet, Brussels. 


\section{Contact information:}

Dragan KOSTIC, PhD, Associate Professor

University of Nis, Faculty of Civil Engineering and Architecture

A. Medvedeva 14, 18000 Nis, Serbia

dragan.kostic@gaf.ni.ac.rs

Vuk MILOSEVIC, M. Arch., Teaching Assistant

University of Nis, Faculty of Civil Engineering and Architecture

A. Medvedeva 14, 18000 Nis, Serbia

vukamer@yahoo.com

Veliborka BOGDANOVIC, PhD, Full Professor

University of Nis, Faculty of Civil Engineering and Architecture

A. Medvedeva 14, 18000 Nis, Serbia

veliborka.bogdanovic@gaf.ni.ac.rs

Miomir VASOV, PhD, Associate Professor

University of Nis, Faculty of Civil Engineering and Architecture

A. Medvedeva 14, 18000 Nis, Serbia

miomir.vasov@gaf.ni.ac.rs

Aleksandar VUCUR, M. Arch, Archineer

ArTech inzenjering

Zvecanska 1a, 11000 Belgrade, Serbia

a.vucur@artech-ing.co.rs 